\title{
Space charge effect in the Multi-channel ionization chamber response signal
}

\author{
A. Nasr $^{1}$ \\ University of Siegen \\ Walter flix strasse 3,57072 Siegen, Germany \\ E-mail: amgad48@gmail.com

\section{H. W. Schenk} \\ University of Siegen \\ Walter flix strasse 3,57072 Siegen, Germany \\ E-mail: schenk@deph.physik.uni-siegen.de
}

\section{A. H. Walenta}

University of Siegen

Walter flix strasse 3,57072 Siegen, Germany

E-mail: walenta@deph.physik.uni-siegen.de

Abstract

The ionization of the gas molecules by X-rays results in electron-ion pair production. The positive ions need several milliseconds to move from the generation position to the cathode. Conversely, the electrons require few microseconds to reach the anode plates. The accumulation of the positive ions inside the active volume, according to their slower drift, leads to space charge effect which has an influence on the signal amplitude.

The Multi-channel ionization chamber is a beam monitor which is developed to measure the intensity of the incoming synchrotron radiation exposing the object and the fluctuation in the beam as a function of time.

The measurements were carried out at the EDR synchrotron beam line at BESSYII. The chamber was filled with a mixture of $90 \%$ Argon and 10\% Methane with a pressure of (5.7 and 10) bar. The applied electric field has been tuned from $400 \mathrm{v} / \mathrm{cm}$ up to $2500 \mathrm{v} / \mathrm{cm}$ at which the space charge effect is clearly observed.

\footnotetext{
$1 \quad$ Speaker
} 
VI European Summer School on Experimental Nuclear Astrophysics Acrireale Italy

September 18-27, 2011 


\section{Introduction:}

The Multi-channel ionization chamber has been built by the detector physics group at Siegen university to monitor the synchrotron radiation beam for the K-edge digital subtraction angiography using synchrotron radiation [1].

The Multi-channel ionization chamber absorbs less than $1 \%$ of the incoming radiation. The chamber is divided into two parts by the anode strip, the depth in each side is $0.5 \mathrm{~cm}$. This will inturn decrease the collection time of positive ions as consequenc of short drift distance the ions move.

\subsection{Space charge effect:}

At high photons flux rate, the most obvious impedance results from the space charge. The space charge has an influence on the signal amplitude of the drifting charges along the signal track. The collection time of the electrons is shorter than the ions by roughly $10^{-3} \mu \mathrm{s}$ due to the mass difference.

The slow drifting positive ion distorts the electric field inside the detector. The distortion of the electric filed affects the drift velocity of the electrons and the ions. This consequently changes the total current density inside the chamber volume [2].

\subsection{Recombination:}

The theoretical model of the recombination in the parallel plate chamber is described by Hübner (1958), Scott and Greening (1961) and Boag(1969)). Boag discussed the general recombination in a parallel plate ionization chamber near the saturation region by considering the varying distribution of electron-ion densities across the space between the chamber plates [3].

The recombination and screening effect due to the space charges are very small in the ionization chamber at low X-ray beam intensity and thus they can be neglected. At high X-ray beam intensity, the effect of the positive ions contribution is significant.

The rate of recombination in the detector can be evaluated quantitatively by considering the $\mathrm{n}+$ and $\mathrm{n}^{-}$for the ion and electron densities respectively, if $\alpha$ is the recombination coefficient, then [4].

$$
\frac{\mathrm{dn}-}{\mathrm{dt}}=-\alpha \mathrm{n}^{+} \mathrm{n}^{-}
$$

\subsection{Saturation current:}

In the ionization chamber, the drift of the positive and negative charges are represented by the applied electric field in a given volume of gas with a continously irradiated from X-ray source. The number of electron ion pairs depend on the gas density, number of photons and energy of the incoming photon flux. The rate of charge will be balanced by the lost of ions pairs from the volume by the recombination and diffusion.

The density of the distribution of the positive ions inside the chamber is called space charge. This leads to a reduction of the electric field inside the chamber which has an influnce on the drift and collection time of the charges. However, The effect is on the signal amplitude within the signal track [2].

The current voltage characteristics of the chamber have been studied using different X-ray intensities by applying different tube currents to increase the photo flux. The electric field is created inside the chamber by applying an external applied high voltage. The perturbation 
analysis of the measured current has been studied by Rossi and Saub (1949), thus leading to the following equation:

$$
-\frac{\nabla \mathrm{I}}{\mathrm{I}}=\frac{\epsilon \mathrm{kT}}{\mathrm{eV}}
$$

where:

$\epsilon=$ is the rario of the average energy of the charge

$\mathrm{I}=$ measured out put current

$\mathrm{K}$ =boltzmann constant

$\mathrm{T}=$ absolute tembrature

$\mathrm{e}=$ electron charge

$\mathrm{V}=$ apllied voltage

The factor ( $€ \mathrm{KT} / \mathrm{e})$ in Eq. 2 is constant and the loss of the current depends mainly on the applied voltage $(\mathrm{V})$ between the tow electrodes.

Figure 1 showes that there is no current flowing in the absence of the applied electric field, since the generated ions and electrons dissappear either by recombination or diffusion in the active volume. As the voltage increases, the electric field begins to drift the positive ion towards the cathod and electrons towards the anode. The measured current increases with the applied voltage due to the increase in the drift velocities of the charges and the reduction of the amount of lost charges. At sufficient high applied voltage, the electric field is high enough to reduce the recombination effect into neglegable value. Therefore, almost all charges are collected in the electrodes. By further increasing of the applied voltage, the output current remains constant beacause the charge collection rate is constant under these conditions. The ionization chamber is conventioally operated where the measureed current is constant which is called saturation current [5].

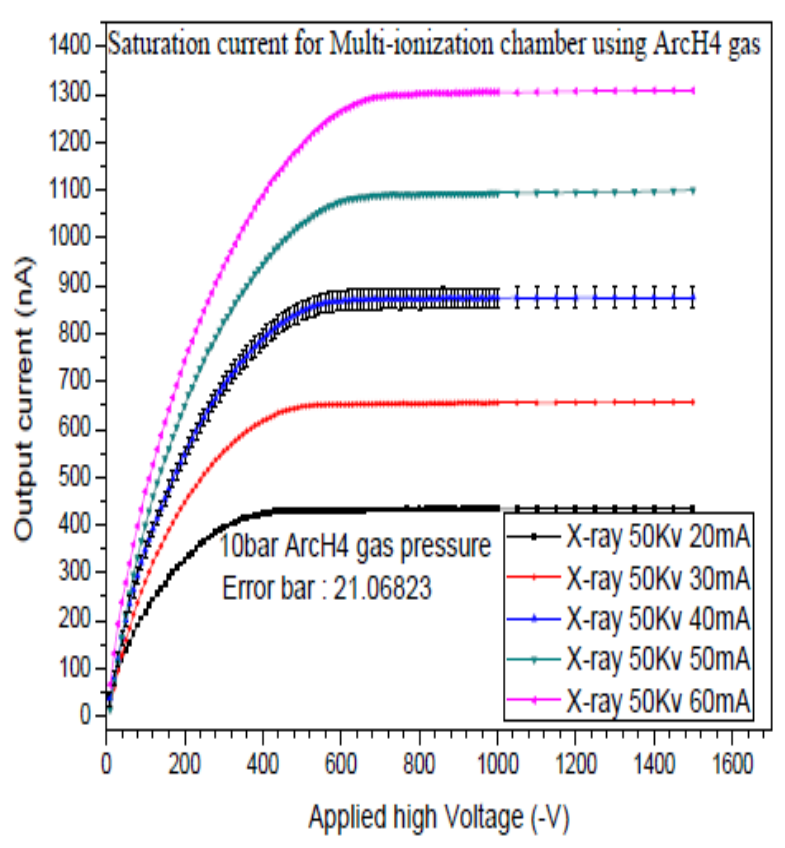

[a]

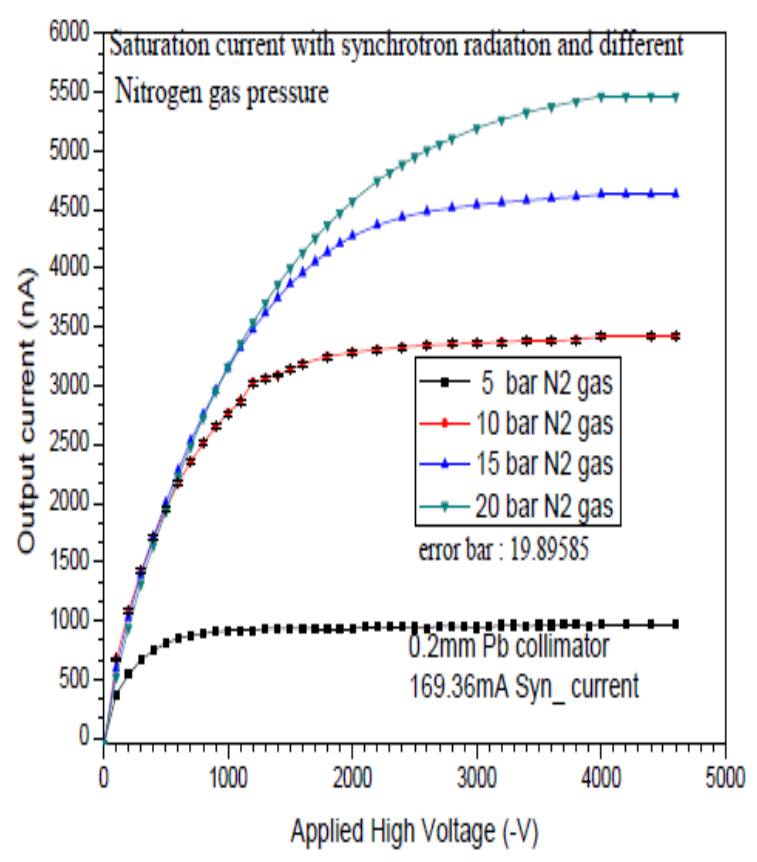

[b]

Figure 1. The current voltage characteristics measurement with different applied voltage with 10bar Nitrogen gas pressure with synchrotron radiation source at EDR beam line (BESSYII) [b] and 10bar ArcH4 gas presure with X-ray tube at Siegen University [a], Showing that the saturation level is increasing by increasing the photon flux intensities. 


\section{The experimental setup:}

The results are obtained by using the Multi-channel ionization chamber built by the detector physics group at Siegen University. More details about the ionization chamber used here can be found in [1]. We filled the chamber with a mixture of 90\% Argon and 10\% Methane with a pressure of $(5,7$ and 10) bar. The measurements were carried out at the EDR synchrotron beam line at BESSYII. The beam size was $(1 \times 1.5) \mathrm{mm}^{2}$. A circular lead chopper with a diameter of 9 $\mathrm{cm}$ and a $1 \mathrm{~mm}$ slit is mounted on a drill machine connected to a power supply. It is used for chopping the synchrotron beam as shown in Fig. 2. Two collimators are placed in front of the chamber: a $(3 \times 50) \mathrm{mm} 2$ horizontal lead collimator is place at the chamber entrance window to protect the lower parts of the chamber from the beam and $(0.2 \times 0.4) \mathrm{mm} 2$ collimator is placed at $4 \mathrm{~cm}$ away from the chamber see Fig. 2.

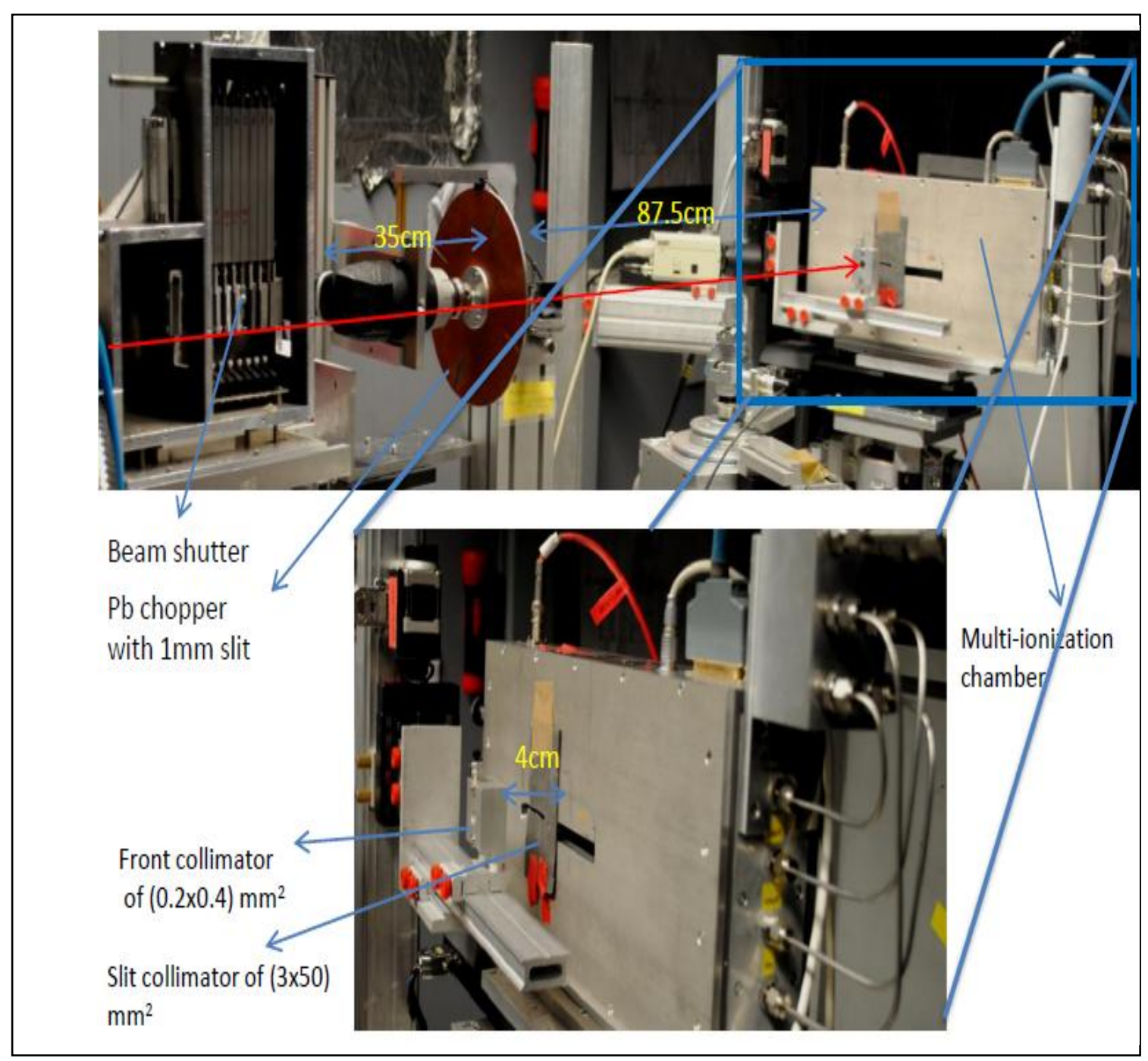

Figure 1. The measurement setup at BESSYII beam line the beam shutter left, Pb chopper at $35 \mathrm{~cm}$ from the shutter, the chamber is placed at $87.5 \mathrm{~cm}$ from the chopper. Magnified image to show the two collimators in front of the chamber. 


\section{Experimental results:}

By using the argon-methane mixture described before, the space charge effect has been investigated by operating the chamber in the recombination region by applying electric field of $400 \mathrm{v} / \mathrm{cm}$.

Strong attenuation in the amplitude signal according to the space charge effect is observed as shown in Fig.3, panel [A]. Operating the chamber in the saturation region by applying an electric field of $2500 \mathrm{v} / \mathrm{cm}$, a plateau is observed at the top of the signal amplitude as shown in Fig.3, panel $[\mathrm{B}]$ where the space charge effect is disappeared, according to the strong electric field which reduces the loss of the charges due to the recombination effect.
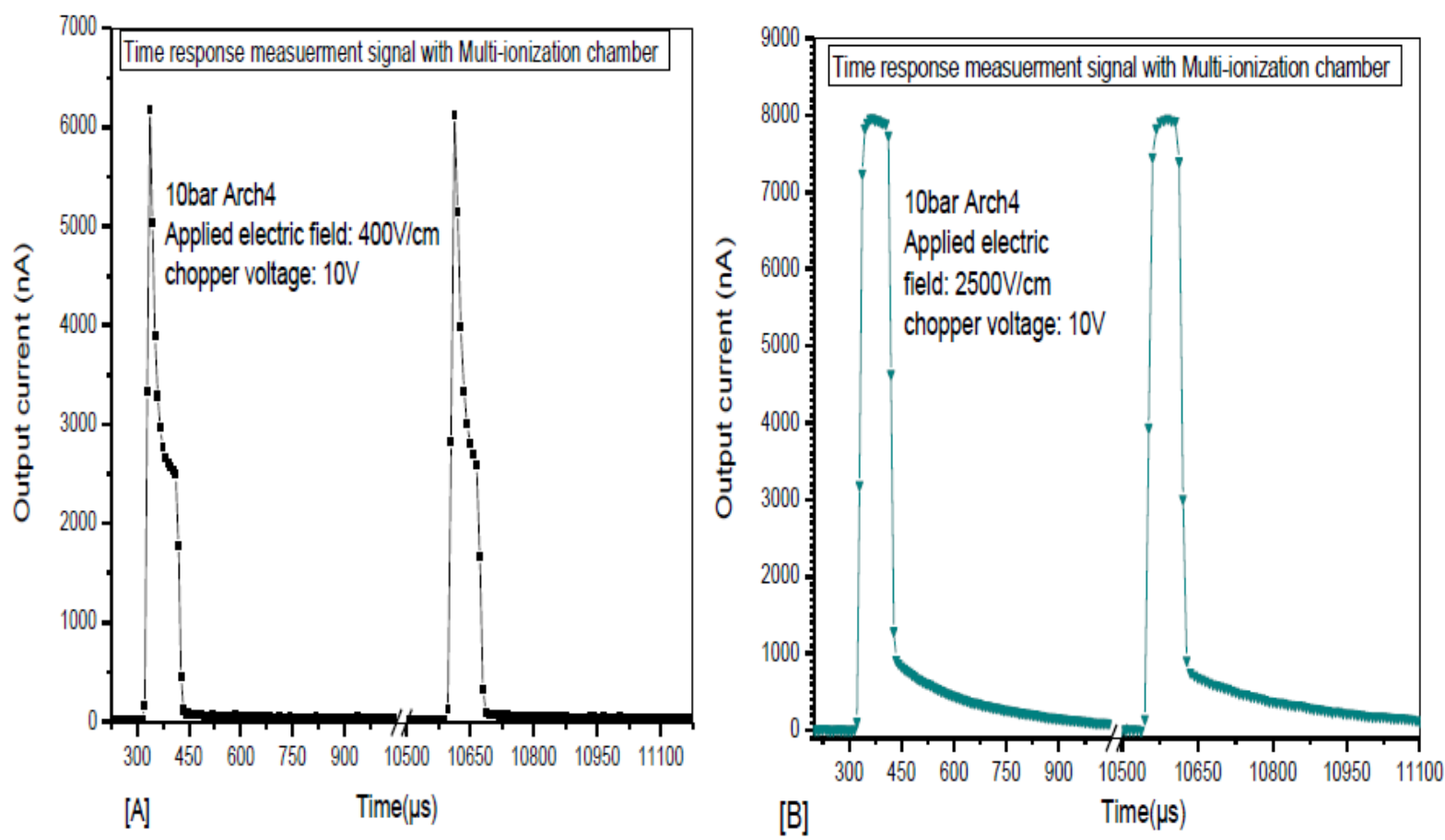

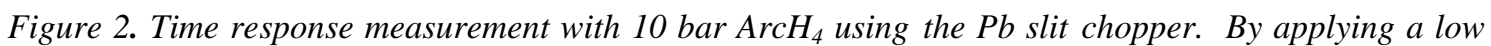
electric field, the slit is open and a fast current signal appears. Immediately it drops down according to the space charge effect which disturbs the electric field and the drift velocity of the charges till it reaches equilibrium and the signal drops down as soon as the chopper closed [A]. By applying a high electric field in the chamber saturation region a time response is measured and a plateau is observed according to the fast drift velocities of the charges as well reduced the recombination of the charges [B].

The measured result of the space charge effect by using argon methane mixture of with a pressure of (5,7 and 10) bar and tuning the applied electric field from $400 \mathrm{v} / \mathrm{cm}$ up to $2500 \mathrm{v} / \mathrm{cm}$ is shown in figures. $(4,5$ and 6$)$ at which the space charge effect is clearly observed. 


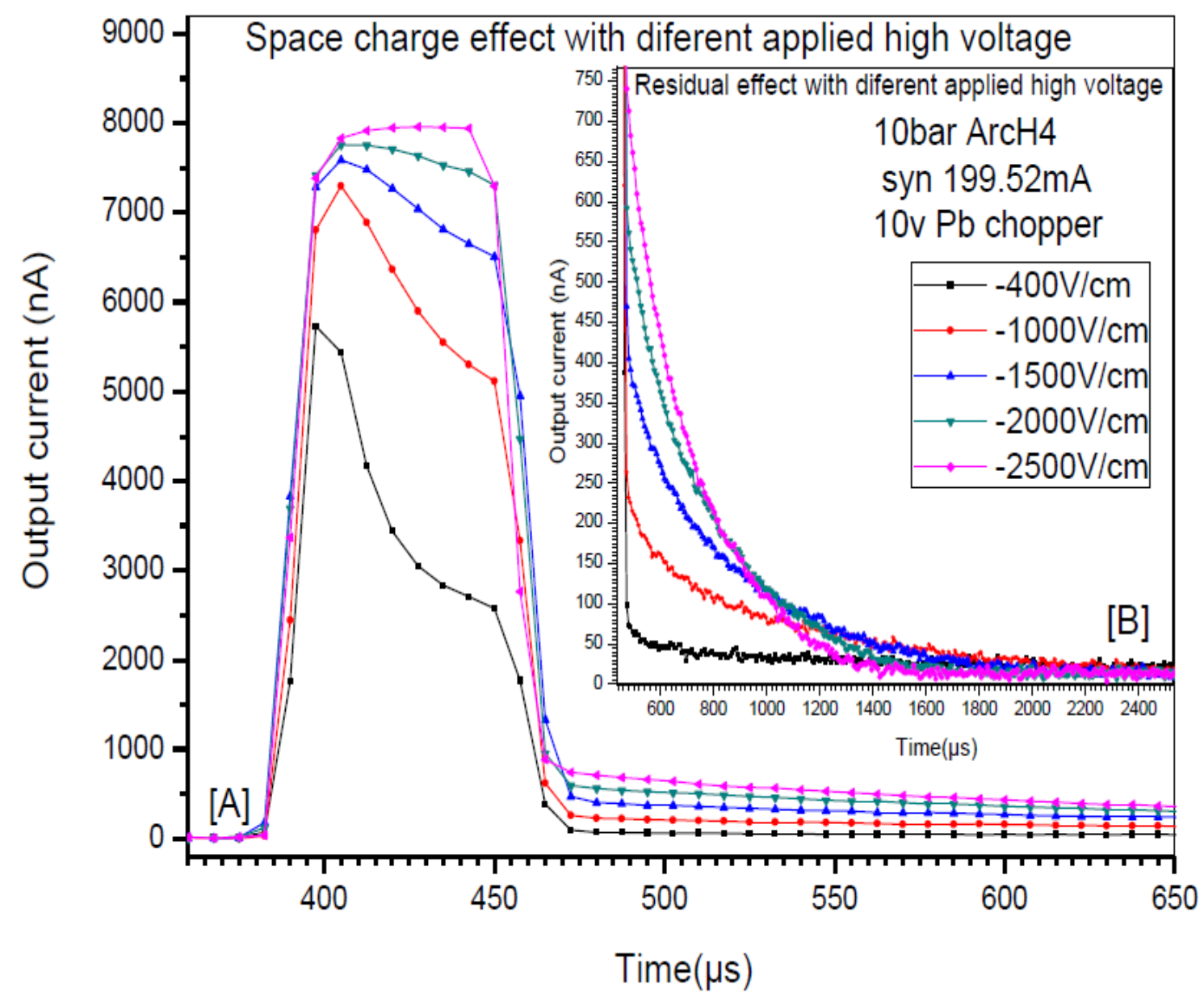

Figure 3. This figures show different measurements of the space charge effect as a function of applied electric field from $400 \mathrm{v} / \mathrm{cm}$ to $2500 \mathrm{v} / \mathrm{cm}$ were the space charge effect decreases with the increasing of the applied electric field [A]. Magnified long tail signal to study the ions collection time [B].

\subsection{Residual offset:}

The long tail signal observed in the measurements as depicted in panel [B] at figure (4, 5 and 6), represents the remaining ions per collection time. At these parts of the measurement the chopper closed and cut out the incoming photon flux. Accordingly there is no new generation of electrons and ions pairs. The remaining electrons inside the active volume are collected at the anode plate in a very short time according to their fast drift velocity compared to the ions drift velocity and remaining only the slow ions inside the active volume of the chamber. The lost of the ions by recombination effect is negligible as all electrons disappeared from the active volume. These ions are continuously drifting toward the cathode and induced a current signal, the ions collection time is in the order of few milliseconds. 


\section{Discussion:}

From the saturation current measurements shown in figure 1 , it is found that the loss in the output current depends mainly on the applied high voltage, according to a measurements of the space charge effect on the signal amplitude at high photon intensity and the relation between the applied high voltage and output current was measured using the Multi-channel ionization chamber.

The time response signal measurements using argon-methane mixture of (90:10) with different pressures shown in figure (4, 5 and 6) provided a quantitative result on the reduction of the signal amplitude and shape due to the space charge accumulation during the drift towards the cathode plate.

When operating the ionization chamber in the recombination region the space charge, recombination and diffusion effects are dominant. It is possible to minimize these effects by operating the ionization chamber in the saturation region.

\section{Acknowledgments:}

I gratefully acknowledge the people who contributed in the design and construction of the Ionization chamber specially the Workshop of the Physics Department at University of Siegen for the technical support and U. Werthenbach, D. Junge, H. Yousef and O. Rübsamen for their help and useful discussion.

\section{References}

[1] V. K. Myalitsin, H. J. Besch, H. W. Schenk, A. H. Walenta, A Multichannel position sensitive ionization chamber for x-ray intensity monitoring. Nucl. Instrum. Methods. A323 (1992)97-103.

[2] A. Andronic, et al, Space charge in drift chambers operated with the Xe,CO2(15\%) mixture, Nucl. Instrum. Methods A 525 (2004) 447-457.

[3] J. W. Boag, General recombination in a standard parallel -plate free air ionization chamber. Int. J. radiat. Phys. Chem.267-285( 1969).

[4] S. W. Massey and H. B. Gilbody, Electronic and ionic impact phenomena, Oxford university press (1974).

[5] Glenn F. knoll, radiation detection and measurement, John Wiley \& Sons, Inc (1999). 


\section{Appendix}

Measurement with applying pressure of 7 bar and 5 bar with argon-methane gas mixture of $(90: 10)$.

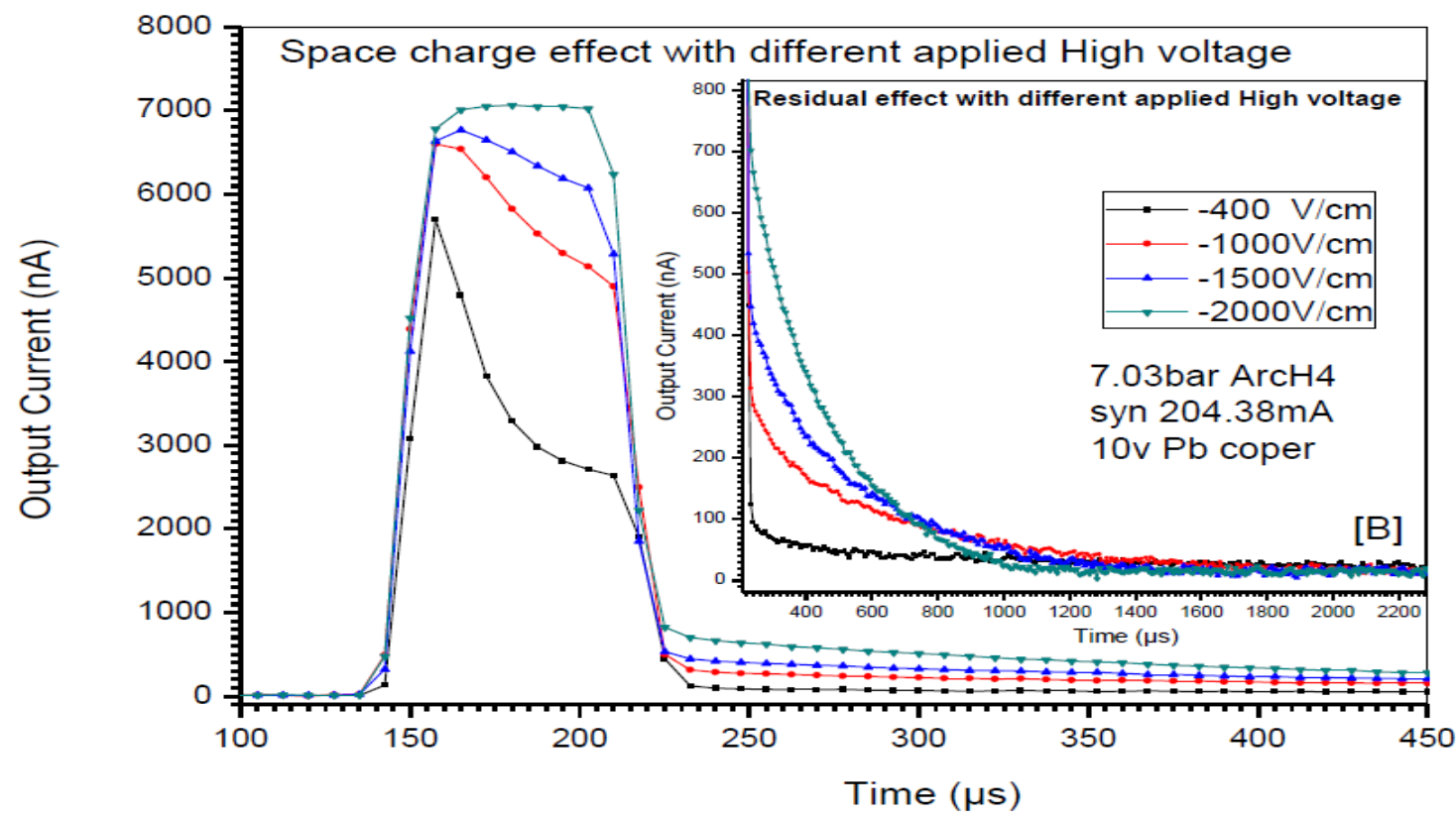

Figure 4. These figures show the time response of 7 bar ArcH4 using setup shown in Fig. 2, the space charge effect observed with applied electric field of $400 \mathrm{v} / \mathrm{cm}, 1000 \mathrm{v} / \mathrm{cm}$ and $1500 \mathrm{v} / \mathrm{cm}$. It reaches the plateau at applied electric field of $2000 \mathrm{v} / \mathrm{cm}$ [A]. Magnified residual signal to study ions collection time $[B]$.

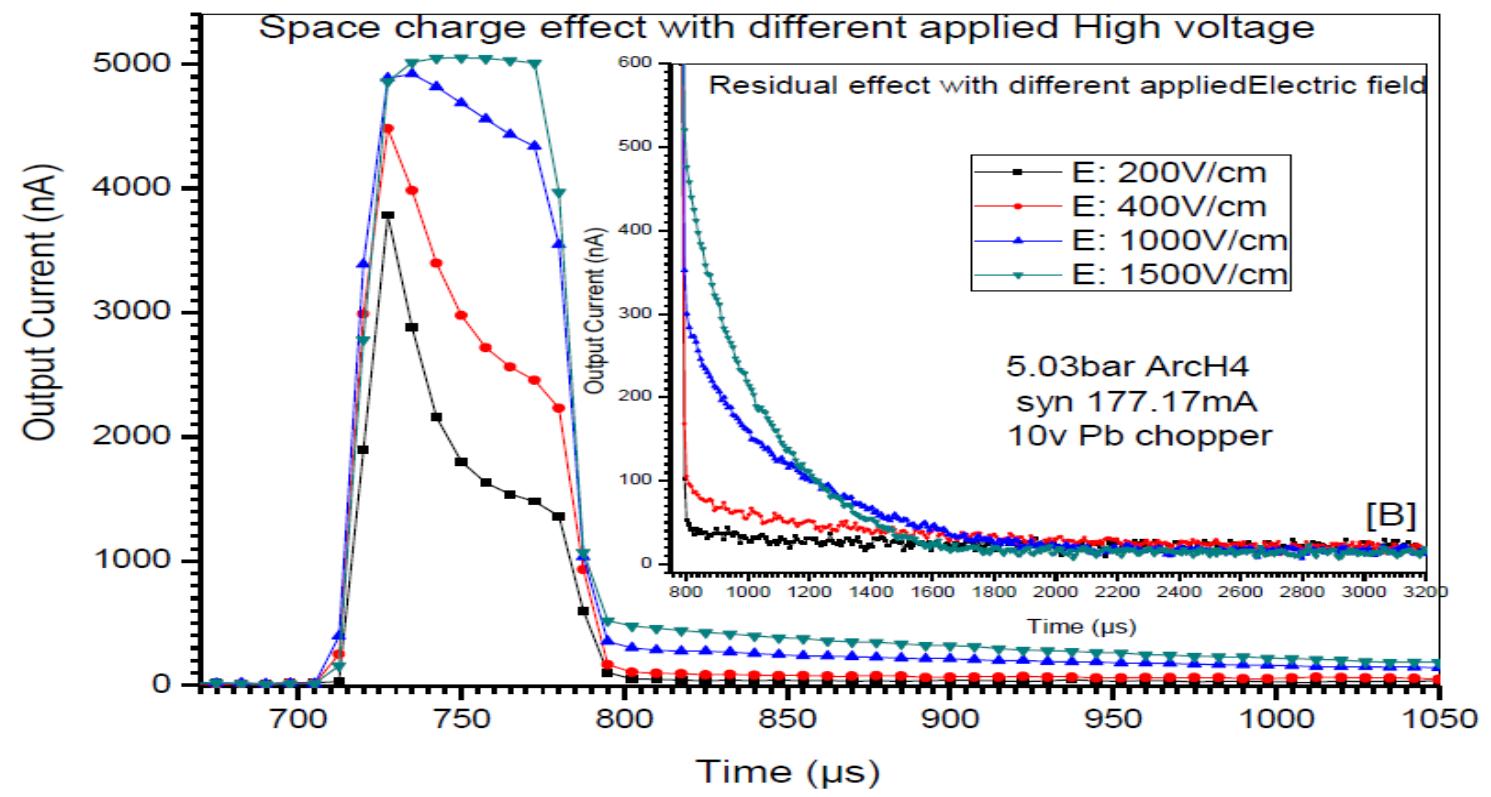

Figure 5. These figures show the time response of 5 bar ArcH4 using setup shown in Fig. 2, the effect of the space charge when applying electric field of $200 \mathrm{v} / \mathrm{cm}, 400 \mathrm{v} / \mathrm{cm}$ and $1000 \mathrm{v} / \mathrm{cm}$ is observed. It reaches the plateau at applied electric field of $1500 \mathrm{v} / \mathrm{cm}$ [A]. Magnified residual signal to study ions collection time [B]. 